\title{
Elevated circulating Gpnmb levels are associated with hyperthyroidism
}

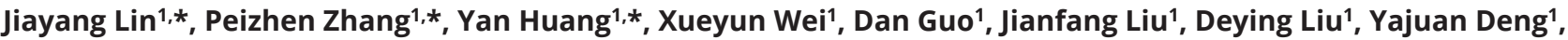 \\ Bingyan Xu', Chensihan Huang ${ }^{1}$, Xiaoyu Yang ${ }^{1}$, Yan Lư ${ }^{2}$, Lijing Jia ${ }^{3}$ and Huijie Zhang 1 \\ 'Department of Endocrinology and Metabolism, Nanfang Hospital, Southern Medical University, Guangzhou, China \\ 2Department of Endocrinology and Metabolism, Zhongshan Hospital, Fudan University, Shanghai, China \\ ${ }^{3}$ Department of Endocrinology, Shenzhen People's Hospital, The First Affiliated Hospital of Southern University of Science and Technology, Shenzhen, \\ Guangdong, China
}

Correspondence should be addressed to L Jia or H Zhang: shenzhenjialijing@126.com or huijiezhang2005@126.com

*(J Lin, P Zhang and Y Huang contributed equally to this work)

\begin{abstract}
Background: Glycoprotein non-metastatic protein B (Gpnmb) has been identified as a new cytokine secreted by hepatocyte that plays an important role in balancing lipid homeostasis and development of obesity and metabolic disorders. However, information is not available regarding the association between circulating Gpnmb and hyperthyroid in humans.

Methods: We measured serum Gpnmb in 180 hyperthyroid patients and 82 healthy subjects that were recruited from the clinic. Of them, 46 hyperthyroid patients received thionamide treatment for 3 months.

Results: Hyperthyroid subjects had higher levels of circulating Gpnmb than healthy controls $(47.8 \pm 10.1 \mathrm{ng} / \mathrm{mL}$ vs $31.0 \pm 4.9 \mathrm{ng} / \mathrm{mL}, P<0.001)$. Subjects with higher levels of serum free triiodothyronine (T3) and free thyroxine (T4) had higher levels of circulating Gpnmb. After thionamide treatment, levels of circulating Gpnmb in hyperthyroid subjects remarkably declined with significant improvement of thyroid function $(P<0.001)$. Furthermore, the change of circulating $G$ pnmb levels was significantly associated with basal metabolic rate (BMR) and thyroid hormones, including free T3 and free T4, adjusting for age, gender, smoking and BMI before thionamide treatment. In multivariable logistic regression analyses, circulating Gpnmb was significantly associated with risks of hyperthyroidism (OR (95\% Cl): $1.44(1.20-1.74), P<0.001)$, adjusted for age, gender, BMI, fasting glucose, HOMA-IR, LDL-cholesterol, ALT and AST. Conclusions: These findings indicate that circulating Gpnmb concentrations are independently associated with hyperthyroid, suggesting that circulating Gpnmb may be a predictor of risk for hyperthyroidism and can be used for therapeutic monitoring.
\end{abstract}
Key Words
- Gpnmb
- thyroid hormone
- hyperthyroidism
- metabolism

\section{Introduction}

Thyroid hormones are essential for both normal development and metabolism. These actions are mediated through its two receptor isoforms, thyroid hormone receptor $\alpha(\mathrm{TR} \alpha)$ and thyroid hormone receptor $\beta$ (TR $\beta$ ) (1). Thyroid hormone has dominant effects on thermogenesis through $\mathrm{TR} \alpha$ that is expressed in the skeletal muscle and adipose tissue, whereas it impacts on lipid metabolism through TR $\beta$ that is mainly expressed in the liver $(2,3,4)$. It is well established that thyroid hormone status correlates with body weight and energy expenditure $(5,6)$. Hyperthyroidism is a condition that excess thyroid hormone promotes a hypermetabolic state (c) 2020 The authors Published by Bioscientifica Ltd

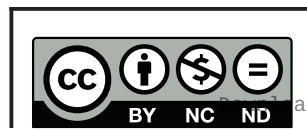

This work is licensed under a Creative Commons Attribution-NonCommercial-NoDerivatives 4.0 Internationab bicense.ifica.com at 04/26/2023 01:29:53PM 
characterized by increased energy expenditure, weight loss, reduced cholesterol, increased lipolysis, and hepatic gluconeogenesis $(7,8,9)$. Recent advances indicate that altered thyroid status can affect circulating levels of cytokines secreted from the liver, including fetuin A, fibroblast growth factor 21 (FGF21), and neuregulin 4 (Nrg4) $(10,11,12)$, suggesting these hepatokines may be involved in the thyroid hormone signaling network to modulate energy homeostasis and lipid metabolism. These observations underscore the importance of hepatokines in cross-talk with thyroid hormone signaling pathway in pathogenesis of hyperthyroidism.

Glycoprotein non-metastatic protein B (Gpnmb) is a highly glycosylated type I transmembrane protein that plays a role in extracellular matrix remodeling in osteoblast and fibroblast differentiation, inflammation and cancer metastasis $(13,14,15,16)$. Gpnmb was initially identified from the osteoblasts, melanoma and various cancer cell lines, macrophages, and dendritic cells, meanwhile, it is also expressed in white adipose tissue (WAT) and liver $(14,17)$. Recently, Gpnmb has been identified as a hepatokine that plays an important role in balancing lipid homeostasis within the whole body via mediating liver-to-WAT communication (18). In human, serum Gpnmb levels were strongly associated with BMI and serves as a risk factor for human obesity and diabetes (18). Akihiro and colleagues suggested that Gpnmb had beneficial impact on hepatic fat accumulation and acted as a biomarker in the development and progression of nonalcoholic steatohepatitis (19).

In addition, previous researchers reported that Gpnmb may regulate autoimmune responses through myeloid-derived suppressor cells or antigen presenting cells (APCs) $(20,21,22)$. It is well established that both cell-mediated and humoral response contribute to the development of autoimmune thyroid diseases (AITD) $(23,24)$. However, information is limited regarding the association between circulating Gpnmb and autoimmune thyroid diseases in humans. In the present study, we aimed to investigate the change of circulating Gpnmb levels in patients with hyperthyroidism during thionamide treatment and explore the association between circulating Gpnmb and hyperthyroidism.

\section{Methods}

\section{Study participants}

A total of 180 adult subjects with initially diagnosis of hyperthyroidism were recruited in Nanfang Hospital of Southern Medical University, Guangzhou, China, from August 2017 to March 2020. Hyperthyroidism was diagnosed by typical clinical presentation, elevated serum thyroid hormone, reduced thyroid stimulating hormone (TSH), and elevated serum TSH receptor antibody (TRAb) levels. All hyperthyroid patients received thionamide treatment. Of them, 46 hyperthyroid patients received thionamide treatment for 3 months and follow-up visit occurred when their TH levels returned to normal range. Additionally, 82 healthy subjects were recruited from physical examination center of Nanfang Hospital of Southern Medical University. All subjects completed a standard questionnaire, including social-demographic status, life style and medical history. Subjects who had cancer, current treatment with antithyroid drugs or systemic corticosteroids, pregnancy, lactation, subacute thyroiditis, abnormal kidney function, and infectious diseases, were excluded.

Written informed consent was obtained from each subject. The study protocol was approved by the Institutional Review Board of Nanfang Hospital of Southern Medical University. The methods were carried out in accordance with the approved guidelines.

\section{Clinical and biochemical measurements}

Anthropometric measurements included height, weight, heart rate, waist circumference, blood pressure, basal metabolic rate (BMR), and BMI (weight in kilograms divided by the square of the height in meters). Waist circumference was measured at the level of the umbilicus. Three measurements were obtained with a non-stretchable tape, and the mean value was used for analysis. Blood pressure (BP) was assessed in triplicate using an electronic sphygmomanometer (OMRON co., Kyoto, Japan). The mean values of the three readings were used for analysis. BMR was estimated by Gale's formula: pulse pressure difference + pulse rate - 111 (25).

All subjects were instructed to fast for $12 \mathrm{~h}$ before screening. Fasting plasma glucose concentrations were measured using the glucose oxidase method. Triglycerides (TG), total cholesterol (TC), and highdensity lipoprotein cholesterol (HDL-c) were measured by enzymatic colorimetric methods with automatic multichannel chemical analyzer (Hitachi 7450). Lowdensity lipoprotein cholesterol (LDL-c) was calculated by Friedewald's formula: TC (mmol/L) - HDL-c (mmol/L) - TG $(\mathrm{mmol} / \mathrm{L}) / 2.2(26)$. Insulin resistance status was assessed using the homeostasis model assessment of insulin resistance (HOMA-IR) according to the following

his work is licensed under a Creative Commons Attribution-NonCommercial-NoDerivatives 4.0 Internationab ficense.ifica.com at 04/26/2023 01:29:53PM 
formula: fasting serum insulin $(\mu \mathrm{U} / \mathrm{mL}) \times$ fasting plasma glucose $(\mathrm{mmol} / \mathrm{L}) / 22.5(27,28)$. Alanine aminotransferase (ALT) and aspartate aminotransferase (AST) were measured using standard enzymatic methods. Total bilirubin (TBIL) and direct bilirubin (DBIL) were measured by diazotization method. Serum insulin, free triiodothyronine (T3), total T3, free thyroxine (T4), total T4, and TSH thyroid peroxide antibody (TPOAb), thyroglobulin antibody (TGAb), and thyrotropin receptor antibody (TRAb) concentrations were measured using electrochemiluminescence immunoassay (Roche Diagnostics).

\section{Serum Gpnmb measurement}

Serum Gpnmb concentrations were measured using ELISA kit (ELH-Osteoactivin, RayBiotech, Inc, Norcross, GA, USA). The assay has been proven to be highly sensitive to human Gpnmb with a sensitivity of $0.045 \mathrm{ng} / \mathrm{mL}$. The intra-assay coefficients of variation were less than $10 \%$, and the interassay coefficients of variation were less than $12 \%$.

\section{Statistical analysis}

All statistical analyses were performed with SAS version 9.4 (SAS Institute Inc). Data are presented as means \pm s.D. or median (interquartile range). Data that were not normally distributed were logarithmically transformed before analysis. The subjects were classified into two groups according to diagnosis of hyperthyroidism. The hyperthyroid subjects were further ranked into four quartiles according to serum Gpnmb levels. The $\chi^{2}$-test and logistic regression models were used for comparison of categorical variables between groups. Analyses of covariance were performed using general linear models (GLM) to test differences in study variables between groups or different quartiles of serum Gpnmb levels. The correlation between changes of serum Gpnmb levels and multiple risk factors was analyzed by Pearson correlation coefficient. Multivariable logistic regression models were used to examine the association of serum Gpnmb levels with risks of hyperthyroidism, adjusted for age, gender, BMI, fasting glucose, HOMA-IR, LDL-c, ALT and AST. Two-sided values of $P<0.05$ were considered statistically significant.

\section{Results}

Table 1 summarizes the mean levels of study variables in subjects with hyperthyroidism and healthy controls.
The mean age of the hyperthyroid patients was $34 \pm 11$ years. Compared with healthy controls, hyperthyroid subjects had higher levels of thyroid hormones, including free T3, free T4, TPOAb, TGAb, and TRAb. as well as lower levels of TSH. Likewise, hyperthyroid subjects had higher levels of BMR, heart rate, ALT, AST, DBIL and TG, and lower levels of TC, diastolic BP, LDL-c and HDL-c compared with healthy controls. BMI, waist circumference, systolic BP, fasting glucose, TBIL and HOMA-IR showed no difference in the two groups. Importantly, hyperthyroid subjects had higher levels of serum Gpnmb than the controls $(47.8 \pm 10.1 \mathrm{ng} / \mathrm{mL}$ vs $31.0 \pm 4.9 \mathrm{ng} / \mathrm{mL}, P<0.001)$.

As shown in Fig. 1, serum Gpnmb levels were significantly correlated with thyroid function, including free T3 $(r=0.660, P<0.001)$, free T4 $(r=0.695, P<0.001)$, TRAb $(r=0.665, P<0.001)$, TPOAb $(r=0.185, P=0.003)$ and TSH $(r=-0.678, P<0.001)$. Serum Gpnmb levels were negatively correlated with BMI $(r=-0.175, P=0.005)$, LDL-c $(r=-0.570, P<0.001)$, HDL-c $(r=-0.263, P<0.001)$ and HOMA-IR $(r=-0.041, P=0.049)$, as shown in Fig. 2 . Additionally, serum Gpnmb levels were positively correlated with BMR $(r=0.589, P<0.001)$. Nevertheless, there were no statistically significant correlation between serum Gpnmb and fasting glucose as well as TC.

Table 2 presents clinical characteristic by quartiles of serum Gpnmb levels, adjusted for age and gender. There were no differences in BMI, SBP, HDL-c, TPOAb and TGAb among the four quartiles of serum Gpnmb levels. Compared to subjects in the lowest quartile of serum Gpnmb levels, those in the highest quartile had higher levels of BMR, heart rate, ALT, AST, free T3, free T4, and TRAb, and lower levels of TC, LDL-c, HDL-c and TSH (all $P<0.05)$. Moreover, ALT, AST, free T3, free T4 and TRAb gradually increased across increasing quartiles of serum Gpnmb levels $(P<0.001)$.

As shown in Supplementary Table 1 see section on supplementary materials given at the end of this article, serum Gpnmb levels were significantly associated with thyroid hormones, including serum free $\mathrm{T} 3$, free $\mathrm{T} 4$, $\mathrm{TSH}, \mathrm{TPOAb}, \mathrm{TGAb}$ and TRAb, adjusting for age, gender, smoking, and BMI. Likewise, serum Gpnmb levels were also significantly associated with liver function and metabolic risk factors, including ALT, AST, systolic BP, diastolic BP, BMR, BMI, TC, LDL-c, and HDL-c. There were no significant associations of serum Gpnmb levels with fasting glucose and HOMA-IR.

The multivariable-adjusted odds ratios (ORs) for the association between serum Gpnmb levels and hyperthyroidism are shown in Table 3. High levels of serum Gpnmb were significantly associated with 
Table 1 Clinical characteristics of hyperthyroid patients and control subjects.

\begin{tabular}{l}
\hline Variables \\
\hline Sample size \\
Age (years) \\
Gender (female, $n \%)$ \\
Current smokers $(n \%)$ \\
BMI $\left(\mathrm{kg} / \mathrm{m}^{2}\right)$ \\
Waist circumference $(\mathrm{cm})$ \\
BMR $(\%)$ \\
Systolic BP $(\mathrm{mmHg})$ \\
Diastolic BP $(\mathrm{mmHg})$ \\
Heart rate $(\mathrm{beats} / \mathrm{mi})$ \\
Triglycerides $(\mathrm{mmol} / \mathrm{L})^{\mathrm{a}}$ \\
Total cholesterol $(\mathrm{mmol} / \mathrm{L})$ \\
LDL-c $(\mathrm{mmol} / \mathrm{L})$ \\
HDL-C $(\mathrm{mmol} / \mathrm{L})$ \\
Fasting glucose $(\mathrm{mmol} / \mathrm{L})$ \\
HOMA-IR \\
ALT $(\mathrm{U} / \mathrm{L})$ \\
AST $(\mathrm{U} / \mathrm{L})$ \\
TBIL $(\mu \mathrm{mol} / \mathrm{L})$ \\
DBIL $(\mu \mathrm{mol} / \mathrm{L})$ \\
Free T3 $(\mathrm{pmol} / \mathrm{L})^{\mathrm{a}}$ \\
Free T4 $(\mathrm{pmol} / \mathrm{L})^{\mathrm{a}}$ \\
TSH $(\mathrm{mIU} / \mathrm{L})^{\mathrm{a}}$ \\
TRAb $(\mathrm{IU} / \mathrm{L})^{\mathrm{a}}$ \\
TGAb $(\mathrm{IU} / \mathrm{mL})^{\mathrm{a}}$ \\
TPOAb $(\mathrm{IU} / \mathrm{mL})^{\mathrm{a}}$ \\
Serum Gpnmb $(\mathrm{ng} / \mathrm{mL})$ \\
\end{tabular}

\begin{tabular}{c}
\hline Overall \\
\hline 262 \\
$33 \pm 10$ \\
$184(70.2)$ \\
$12(4.5)$ \\
$20.8 \pm 3.0$ \\
$74.6 \pm 8.6$ \\
$33.9 \pm 24.8$ \\
$122 \pm 14$ \\
$73 \pm 10$ \\
$96 \pm 20$ \\
$0.83(0.64-1.06)$ \\
$4.09 \pm 0.99$ \\
$2.35 \pm 0.85$ \\
$1.32 \pm 0.32$ \\
$5.06 \pm 0.63$ \\
$1.56(1.03-2.27)$ \\
$29.9 \pm 16.0$ \\
$25.3 \pm 9.5$ \\
$12.9 \pm 5.5$ \\
$4.6 \pm 2.2$ \\
$15.6(5.4-30.8)$ \\
$39.4(16.3-71.6)$ \\
$0.009(0.004-1.249)$ \\
$8.8(0.3-22.5)$ \\
$18.5(9.7-124.4)$ \\
$11.7(4.9-112.7)$ \\
$42.5 \pm 11.8$ \\
\end{tabular}

\begin{tabular}{c}
\hline Hyperthyroid \\
\hline 180 \\
$34 \pm 11$ \\
$125(69.4)$ \\
$12(6.7)$ \\
$20.5 \pm 3.2$ \\
$74.7 \pm 8.4$ \\
$44.6 \pm 22.3$ \\
$123 \pm 15$ \\
$72 \pm 11$ \\
$105 \pm 17$ \\
$0.88(0.70-1.11)$ \\
$3.59 \pm 0.81$ \\
$1.87 \pm 0.61$ \\
$1.26 \pm 0.33$ \\
$5.13 \pm 0.64$ \\
$1.37(0.96-2.16)$ \\
$35.7 \pm 14.3$ \\
$27.4 \pm 10.4$ \\
$13.0 \pm 5.9$ \\
$4.9 \pm 2.5$ \\
$26.2(15.1-89.2)$ \\
$63.9(38.7-83.5)$ \\
$0.006(0.003-0.009)$ \\
$17.2(7.2-29.8)$ \\
$26.0(11.5-243.9)$ \\
$26.4(5.9-192.5)$ \\
$47.8 \pm 10.1$ \\
\end{tabular}

\begin{tabular}{c}
\hline Controls \\
\hline 82 \\
$31 \pm 9$ \\
$59(72.0)$ \\
$0(0)$ \\
$21.1 \pm 2.7$ \\
$74.4 \pm 9.0$ \\
$11.5 \pm 10.9$ \\
$119 \pm 12$ \\
$75 \pm 8$ \\
$78 \pm 8$ \\
$0.74(0.61-0.97)$ \\
$4.77 \pm 0.79$ \\
$2.98 \pm 0.69$ \\
$1.40 \pm 0.30$ \\
$4.97 \pm 0.60$ \\
$1.79(1.23-2.36)$ \\
$17.8 \pm 12.2$ \\
$21.1 \pm 5.3$ \\
$12.8 \pm 4.7$ \\
$3.9 \pm 1.3$ \\
$5.0(4.7-5.4)$ \\
$15.0(13.8-16.4)$ \\
$1.828(1.262-2.851)$ \\
$0.3(0.3-0.3)$ \\
$12.8(8.4-21.5)$ \\
$6.9(3.3-12.0)$ \\
$31.0 \pm 4.9$ \\
\end{tabular}

\begin{tabular}{c}
\hline P-value $^{\mathbf{b}}$ \\
\hline- \\
$\mathbf{0 . 0 4 2}$ \\
0.681 \\
$\mathbf{0 . 0 1 7}$ \\
0.051 \\
0.895 \\
$<0.001$ \\
0.180 \\
$\mathbf{0 . 0 1 3}$ \\
$<0.001$ \\
$\mathbf{0 . 0 3 0}$ \\
$<\mathbf{0 . 0 0 1}$ \\
$<\mathbf{0 . 0 0 1}$ \\
$\mathbf{0 . 0 0 3}$ \\
0.203 \\
0.052 \\
$<0.001$ \\
$<0.001$ \\
0.805 \\
$<\mathbf{0 . 0 0 1}$ \\
$<\mathbf{0 . 0 0 1}$ \\
$<0.001$ \\
$<\mathbf{0 . 0 0 1}$ \\
$<\mathbf{0 . 0 0 1}$ \\
$<\mathbf{0 . 0 0 1}$ \\
$<\mathbf{0 . 0 0 1}$ \\
$<\mathbf{0 . 0 0 1}$ \\
\hline
\end{tabular}

Data are presented as the mean \pm S.D. or median (interquartile range).

${ }^{a}$ Analysis performed on log-transformed data; badjusted for age and gender.

ALT, alanine transaminase; AST, aspartate aminotransferase; BMR, basal metabolic rate; BP, blood pressure; DBIL, direct bilirubin; Gpnmb, glycoprotein non metastatic melanoma protein B; HOMA-IR, homeostasis model assessment of insulin resistance; LDL-c, low density lipoprotein cholesterol; TBIL, total bilirubin; T3, triiodothyronine; T4, thyroxine; TSH, thyroid stimulating hormone; TPOAb, thyroid peroxide antibody; TGAb, thyroglobulin antibody; TRAb, thyrotropin receptor antibody.

hyperthyroidism (OR (95\% CI):1.41 (1.30-1.54), P<0.001), after adjusting for age and gender. After further adjusting for BMI, fasting glucose, HOMA-IR, LDL-c, ALT and AST, the ORs for hyperthyroidism remained significant (OR (95\% CI):1.44 (1.20-1.74), P < 0.001). Additionally, LDL-c, ALT and AST were also significantly association with hyperthyroidism $(P<0.001)$.

Table 4 summarizes the mean levels of study variables after thionamide treatment in hyperthyroid subjects. After 3-month thionamide treatment, thyroid hormones including free $\mathrm{T} 3$, free $\mathrm{T} 4$, total $\mathrm{T} 3$, total $\mathrm{T} 4$, and $\mathrm{TSH}$, were significantly improved. Additionally, hyperthyroid subjects also showed significant improvement with liver function and metabolic risk factors, including systolic BP, heart rate, BMR, fasting glucose and cholesterol levels. Of note, serum Gpnmb levels were dramatically decreased from $47.6 \pm 8.6$ to $36.8 \pm 5.5 \mathrm{ng} / \mathrm{mL}$ after treatment $(P<0.001)$.

Table 5 presents results of linear regression analyses of change of serum Gpnmb levels on metabolic risk factors and thyroid hormones over 3-month thionamide treatment. In multivariable linear regression analyses, change of serum Gpnmb levels was significantly associated with thyroid hormones, adjusting for age, gender, smoking and BMI after 3-month thionamide treatment. Besides, change of serum Gpnmb levels was also significantly associated with BMR. However, there were no significant associations between changes of serum Gpnmb levels and BMI, waist circumference, BP, fasting glucose, HOMA-IR, lipid profiles, aminotransferase and thyroid antibodies.

\section{Discussion}

In the present study, we found that circulating Gpnmb concentrations are significantly elevated in subjects with hyperthyroidism and markedly declined after thionamide treatment. In addition, circulating Gpnmb concentrations were independently associated with hyperthyroidism and metabolic status, after adjustment

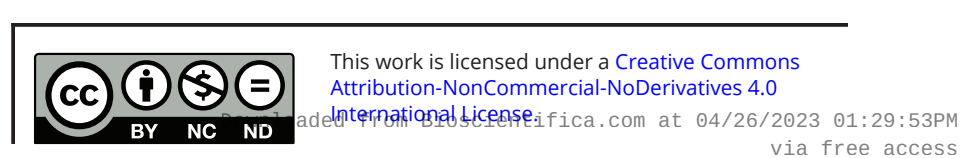



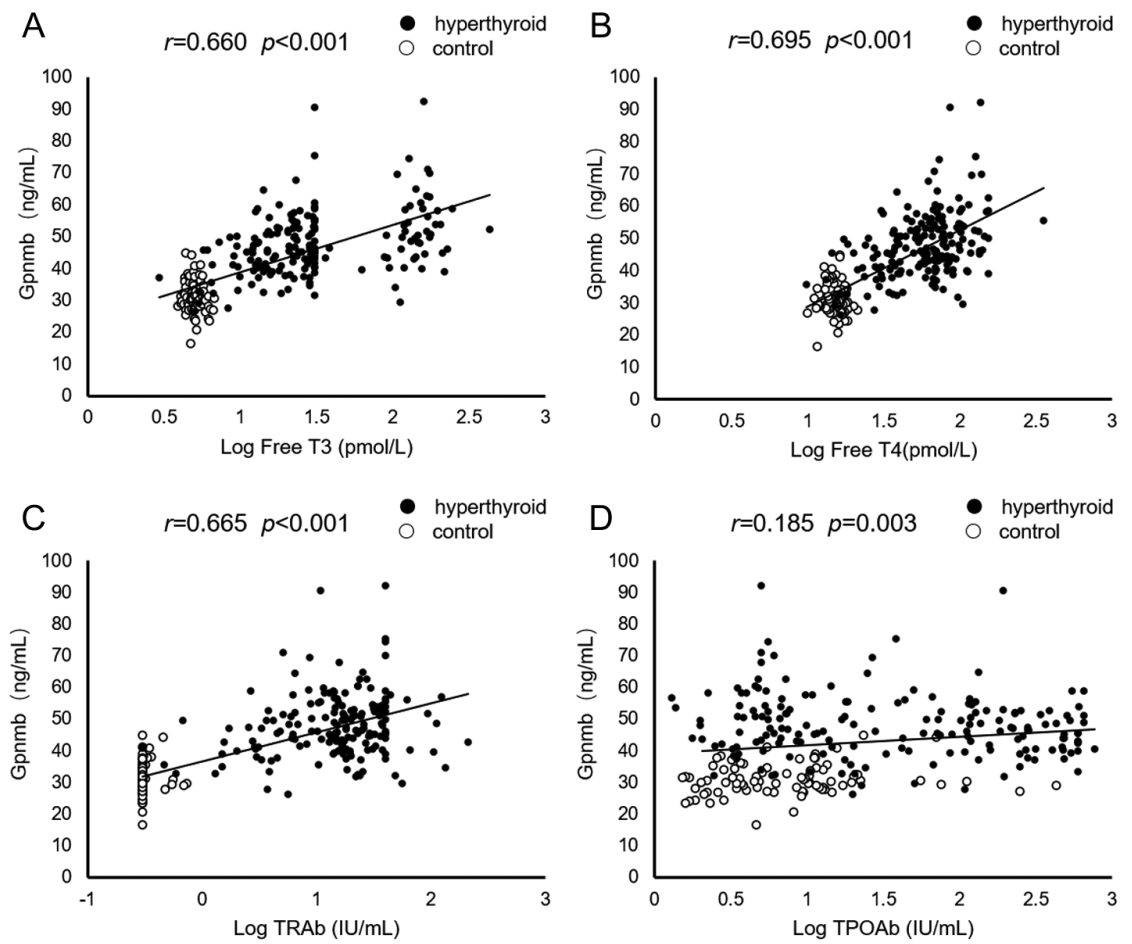

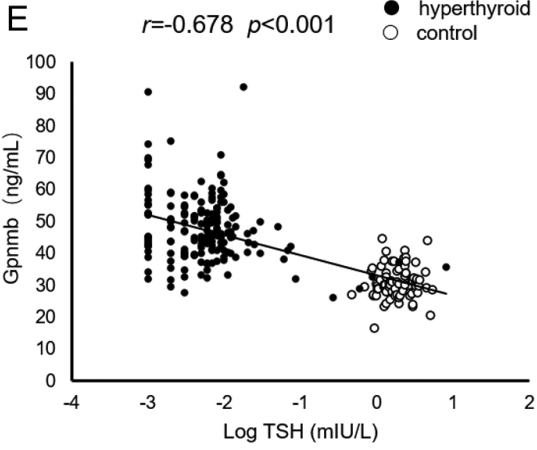

for potential confounders. These findings indicate that circulating Gpnmb could be a prognostic biomarker in the pathogenesis of hyperthyroidism.

It has been well documented that hyperthyroidism is characterized by increased resting energy expenditure, reduced cholesterol levels, increased lipolysis and gluconeogenesis $(7,8,9)$. Thyroid hormone modulates hepatic lipogenesis and bile acid homeostasis through down-regulating sterol regulatory element-binding protein 1-c (SREBP1-c) and induction of CPY7A1 gene expression as well as cross-talk with other nuclear receptors, such as peroxisome proliferator-activated receptor $\alpha$ (PPAR $\alpha)$ and liver X receptor (LXR) pathway $(29,30,32)$. Gong et al. reported that inhibition of the hepatic sterol regulatory element-binding protein pathway leads to increased transcription of Gpnmb and promotes processing of its secretion (18). In the present study, we found that serum Gpnmb levels were significantly elevated in subjects

\section{Figure 1}

Correlation of serum Gpnmb levels with thyroid hormones. (A) Correlation of serum Gpnmb levels with serum levels of free T3; (B) correlation of serum Gpnmb levels with serum levels of free T4; (C) correlation of serum Gpnmb levels with serum levels of TRAb; (D) correlation of serum Gpnmb levels with serum levels of TPOAb; $(E)$ correlation of serum Gpnmb levels with serum levels of TSH. $T 3$, triiodothyronine; $T 4$, thyroxine; TRAb, thyrotropin receptor antibody; TPOAb, thyroid peroxide antibody; TSH, thyroid stimulating hormone. with hyperthyroidism and dramatically declined after thionamide treatment. Furthermore, our data indicated that circulating Gpnmb concentrations were significantly associated with levels of serum free T3, free T4 and TSH, suggesting that excess thyroid hormone would result in an increased secretion of Gpnmb from the liver.

Previous study has shown that serum Gpnmb levels strongly correlated with BMI in obese adults and could act as a risk factor for human obesity and diabetes (18). Gpnmb stimulates lipogenesis in WAT through binding its receptor CD44 and exacerbates diet-induced obesity and insulin resistance in animal model (18). In contrast, our data demonstrated that serum Gpnmb levels were inversely associated with metabolic risk factors, including BMI, TC, LDL-c, and HDL-c. Furthermore, our findings indicated that serum Gpnmb levels were positively associated with BMR in hyperthyroid patients. Similarly, previous studies also reported that several circulating cytokines including 
A
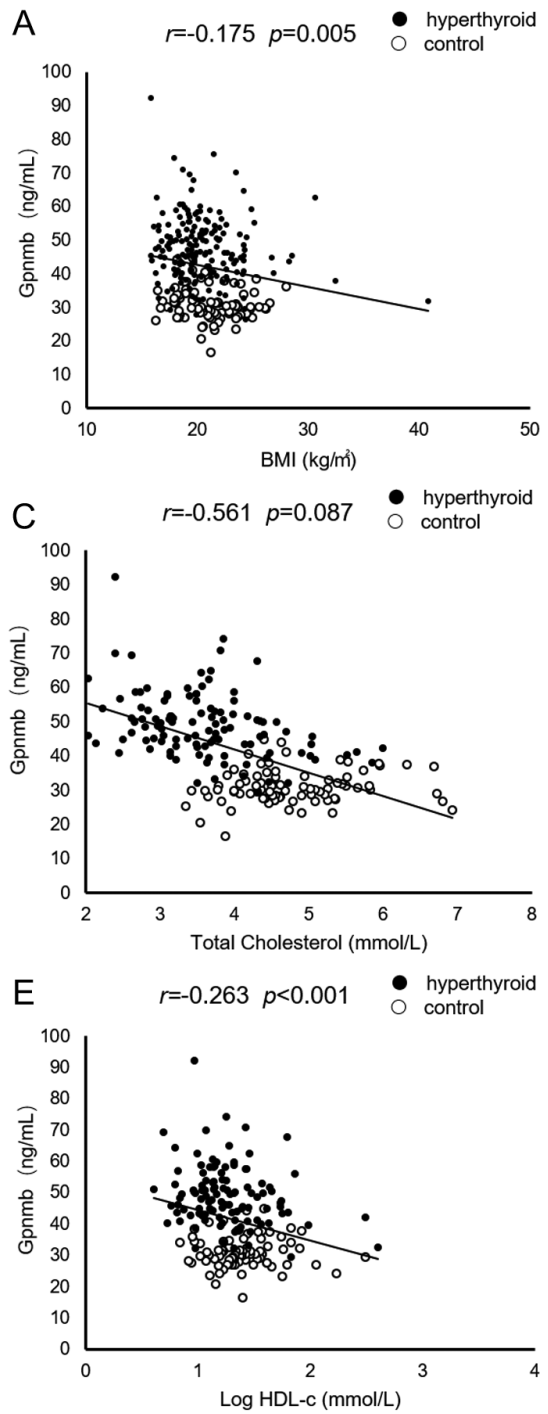

G $\quad r=-0.041 p=0.049 \quad \stackrel{\bullet}{\circ}$ conpertrol

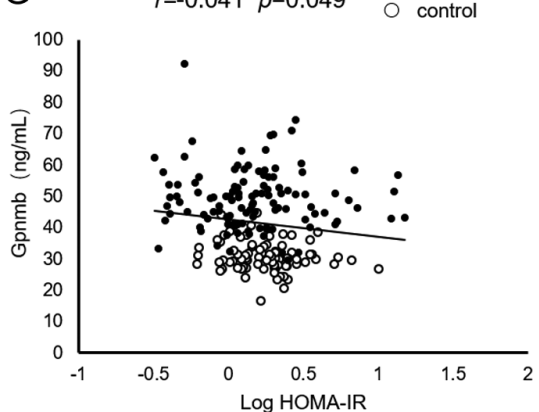

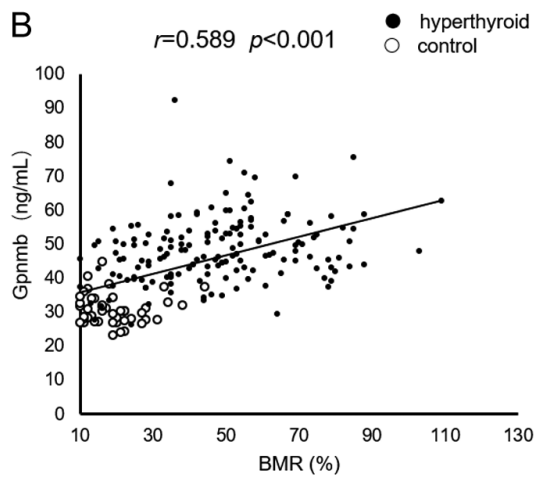
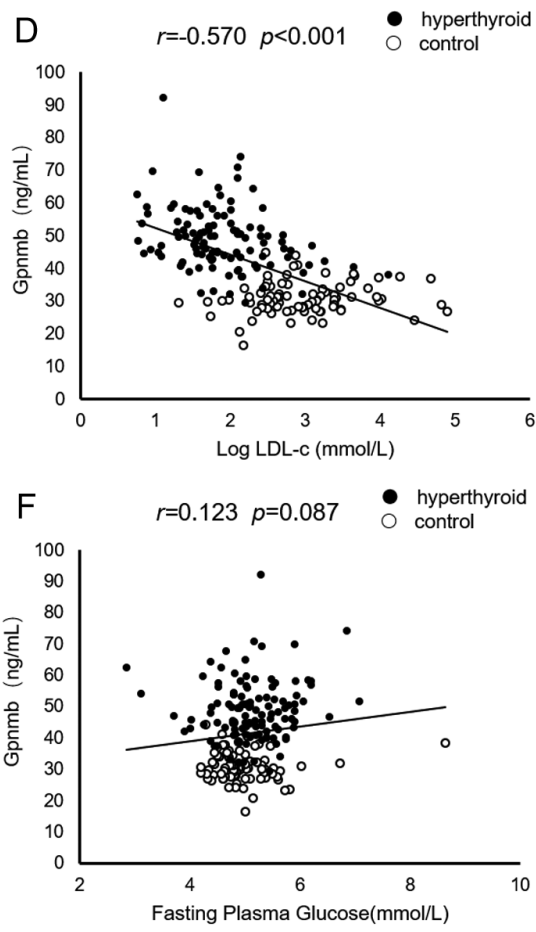

Figure 2

Correlation of serum Gpnmb levels with metabolic risk factors. (A) Correlation of serum Gpnmb levels with BMI; (B) correlation of serum Gpnmb levels with BMR; (C) correlation of serum Gpnmb levels with serum levels of total cholesterol; (D) correlation of serum Gpnmb levels with serum levels of LDL-C; (E) correlation of serum Gpnmb levels with serum levels of HDL-c; (F) correlation of serum Gpnmb levels with serum levels of fasting glucose; $(G)$ correlation of serum Gpnmb levels with HOMA-IR. BMR, basal metabolic rate; $L D L-c$, low density lipoprotein cholesterol; HDL-c, high density lipoprotein cholesterol; HOMA-IR, homeostasis model assessment of insulin resistance.
FGF21 and Nrg4, were correlated with BMR and metabolic risk factors in patients with hyperthyroidism $(10,11,12)$. However, the relationship between Gpnmb and energy expenditure is unclear. Preliminary observation suggests that Gpnmb appears to negatively regulate thermogenesis of brown fat tissue in the obese setting (18). Altogether, these findings support the notion Gpnmb serves as a feedback hormonal signal that is responsive to increased energy expenditure in hyperthyroid patients. Perspective cohort studies are needed to confirm these finding and elucidate potential metabolic mechanisms connecting Gpnmb and thyroid hormones.

In addition, our study indicated that circulating Gpnmb concentrations were significantly associated with levels 
Table 2 Clinical characteristics by quartiles of serum Gpnmb levels in hyperthyroid subjects.

\begin{tabular}{|c|c|c|c|c|c|}
\hline \multirow[b]{2}{*}{ Variables } & \multicolumn{4}{|c|}{ Serum Gpnmb level } & \multirow{2}{*}{$\begin{array}{c}\text { P-value for } \\
\text { trend }^{\mathbf{b}}\end{array}$} \\
\hline & Quartile 1 & Quartile 2 & Quartile 3 & Quartile 4 & \\
\hline Sample size & 44 & 45 & 46 & 45 & \\
\hline Serum Gpnmb (ng/mL) & $36.5 \pm 4.0$ & $43.9 \pm 1.9^{d}$ & $49.8 \pm 2.2^{d}$ & $60.7 \pm 8.8^{d}$ & $<0.001$ \\
\hline Age (years) & $32 \pm 9$ & $35 \pm 11$ & $36 \pm 12$ & $32 \pm 10$ & 0.180 \\
\hline Gender (female $n, \%$ ) & $31(70.5)$ & $31(68.9)$ & $32(69.6)$ & $31(68.9)$ & 0.998 \\
\hline BMI $\left(\mathrm{kg} / \mathrm{m}^{2}\right)$ & $21.1 \pm 4.4$ & $20.8 \pm 3.0$ & $19.8 \pm 2.2^{c}$ & $20.1 \pm 2.7$ & 0.159 \\
\hline Waist circumference $(\mathrm{cm})$ & $76.0 \pm 9.8$ & $76.2 \pm 9.5$ & $73.7 \pm 7.4$ & $73.1 \pm 6.1$ & 0.217 \\
\hline $\operatorname{BMR}(\%)$ & $35.0 \pm 21.3$ & $43.9 \pm 23.2$ & $43.3 \pm 21.9^{c}$ & $55.9 \pm 18.2^{d}$ & $<0.001$ \\
\hline Heart rate (beats/min) & $97 \pm 17$ & $106 \pm 18^{d}$ & $104 \pm 16^{c}$ & $112 \pm 15^{d}$ & $<0.001$ \\
\hline Systolic BP (mmHg) & $121 \pm 14$ & $123 \pm 19$ & $121 \pm 16$ & $126 \pm 12^{c}$ & 0.268 \\
\hline Diastolic BP (mmHg) & $72 \pm 13$ & $73 \pm 11$ & $71 \pm 11$ & $72 \pm 9$ & 0.652 \\
\hline Triglycerides (mmol/L) & $0.84(0.68-1.01)$ & $0.95(0.74-1.22)$ & $0.87(0.68-1.11)$ & $0.82(0.72-1.02)$ & 0.607 \\
\hline Total cholesterol (mmol/L) & $4.08 \pm 0.87$ & $3.65 \pm 0.95^{c}$ & $3.54 \pm 0.65^{d}$ & $3.21 \pm 0.60^{c}$ & 0.001 \\
\hline LDL-c (mmol/L) & $2.21 \pm 0.68$ & $1.87 \pm 0.67^{c}$ & $1.89 \pm 0.52^{c}$ & $1.62 \pm 0.48^{d}$ & 0.003 \\
\hline HDL-c (mmol/L) & $1.41 \pm 0.39$ & $1.28 \pm 0.39$ & $1.22 \pm 0.26$ & $1.19 \pm 0.26^{c}$ & 0.133 \\
\hline Fasting glucose (mmol/L) & $5.12 \pm 0.42$ & $5.12 \pm 0.60$ & $5.21 \pm 0.63$ & $5.06 \pm 0.82$ & 0.867 \\
\hline HOMA-IR & $1.37(1.03-2.27)$ & $1.28(0.87-2.84)$ & $1.43(0.96-2.00)$ & $1.36(0.60-2.00)$ & 0.691 \\
\hline ALT (U/L) & $27.9 \pm 13.0$ & $36.3 \pm 14.4^{d}$ & $38.1 \pm 12.5^{d}$ & $40.1 \pm 14.7^{d}$ & $<0.001$ \\
\hline AST (U/L) & $20.9 \pm 6.9$ & $27.8 \pm 9.2^{d}$ & $29.6 \pm 10.2^{d}$ & $30.8 \pm 11.7^{d}$ & $<0.001$ \\
\hline Free T3 $(\mathrm{pmol} / \mathrm{mL})^{\mathrm{a}}$ & $18.2(9.8-28.5)$ & $27.1(14.2-33.4)^{c}$ & $24.8(15.8-116.3)^{d}$ & $30.8(22.7-155.0)^{d}$ & $<0.001$ \\
\hline Free T4 (pmol/mL) ${ }^{a}$ & $40.6(28.0-69.1)$ & $64.6(36.4-80.8)^{c}$ & $57.9(42.3-87.3)^{c}$ & $72.7(60.8-102.7)^{d}$ & $<0.001$ \\
\hline $\mathrm{TSH}(\mathrm{mlU} / \mathrm{L})^{\mathrm{a}}$ & $0.006(0.003-0.011)$ & $0.006(0.003-0.009)$ & $0.006(0.003-0.008)$ & $0.005(0.001-0.009)^{d}$ & 0.003 \\
\hline $\operatorname{TRAb}(\mathrm{IU} / \mathrm{L})^{\mathrm{a}}$ & $14.2(2.9-22.5)$ & $17.8(10.5-29.7)^{c}$ & $17.8(9.0-25.8)$ & $20.3(11.1-39.4)^{d}$ & $<0.001$ \\
\hline TGAb $(I U / m L)^{a}$ & $19.7(10.4-182.1)$ & $37.8(11.5-394.3)$ & $42.3(16.0-272.2)$ & $16.6(8.4-234.3)$ & 0.112 \\
\hline TPOAb (IU/mL)a & $33.2(7.6-248.2)$ & $57.0(6.7-275.9)$ & $66.3(7.3-231.0)$ & $10.2(4.9-90.6)^{c}$ & 0.068 \\
\hline
\end{tabular}

Data are presented as the mean \pm S.D. or median (interquartile range).

${ }^{a}$ Analysis performed on log-transformed data; ${ }^{b}$ Adjusted for age and gender; ${ }^{c} P<0.05$ compared with Q1 of serum Gpnmb level; $\mathrm{d} P<0.01$ compared with Q1 of serum Gpnmb level.

ALT, alanine transaminase; AST, aspartate aminotransferase; BMR, basal metabolic rate; BP, blood pressure; Gpnmb, glycoprotein non metastatic melanoma protein B.; HOMA-IR, homeostasis model assessment of insulin resistance; LDL-c, low density lipoprotein cholesterol; T3, triiodothyronine; T4, thyroxine; TSH, thyroid stimulating hormone; TPOAb, thyroid peroxide antibody; TGAb, thyroglobulin antibody; TRAb, thyrotropin receptor antibody.

Table 3 Odds ratios of hyperthyroidism according to serum Gpnmb levels.

\begin{tabular}{|c|c|c|c|}
\hline & OR & $95 \% \mathrm{Cl}$ & P-value \\
\hline \multicolumn{4}{|l|}{ Model 1} \\
\hline Serum Gpnmb (ng/mL) & 1.41 & $1.29-1.53$ & $<0.001$ \\
\hline \multicolumn{4}{|l|}{ Model 2} \\
\hline Age (years) & 0.98 & $0.94-1.03$ & 0.446 \\
\hline Gender (female vs male) & 1.35 & $0.54-3.35$ & 0.520 \\
\hline Serum Gpnmb (ng/mL) & 1.41 & $1.30-1.54$ & $<0.001$ \\
\hline \multicolumn{4}{|l|}{ Model 3} \\
\hline Age & 1.01 & $0.93-1.10$ & 0.837 \\
\hline Gender (female vs male) & 1.19 & $0.93-8.89$ & 0.867 \\
\hline $\mathrm{BMI}\left(\mathrm{kg} / \mathrm{m}^{2}\right)$ & 0.83 & $0.55-1.26$ & 0.390 \\
\hline Fasting glucose (mmol/L) & 2.52 & $0.71-8.97$ & 0.152 \\
\hline HOMA-IR & 0.72 & $0.41-1.25$ & 0.239 \\
\hline LDL-C (mmol/L) & 0.08 & $0.02-0.33$ & $<0.001$ \\
\hline $\operatorname{ALT}(U / L)$ & 1.18 & $1.07-1.31$ & 0.001 \\
\hline AST (U/L) & 0.81 & $0.67-0.97$ & 0.024 \\
\hline Serum Gpnmb (ng/mL) & 1.44 & $1.20-1.74$ & $<0.001$ \\
\hline
\end{tabular}

Model 1: without adjustment. Model 2: adjusted age, gender. Model 3: adjusted for model $2+$ BMI, fasting glucose, HOMA-IR, LDL-C, ALT and AST. ALT, alanine transaminase; AST, aspartate aminotransferase; Gpnmb, glycoprotein non metastatic melanoma protein B; HOMA-IR, homeostasis model assessment of insulin resistance; LDL-c, low density lipoprotein cholesterol; OR, odds ratio. Bold indicates statistical significance $(P<0.05)$.

https://ec.bioscientifica.com https://doi.org/10.1530/EC-20-0240 (c) 2020 The authors Published by Bioscientifica Ltd

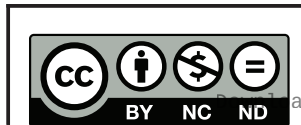

This work is licensed under a Creative Commons Attribution-NonCommercial-NoDerivatives 4.0 International License.ifica com at 04/26/2023 01:29:53PM 
Table 4 Serum Gpnmb levels before and after thionamide treatment in hyperthyroid patients.

\begin{tabular}{|c|}
\hline Variables \\
\hline Sample size \\
\hline Age (years) \\
\hline Gender (female, $n \%$ ) \\
\hline Current smokers ( $n \%)$ \\
\hline $\mathrm{BMI}\left(\mathrm{kg} / \mathrm{m}^{2}\right)$ \\
\hline Waist circumference $(\mathrm{cm})$ \\
\hline $\operatorname{BMR}(\%)$ \\
\hline Heart rate (beats/min) \\
\hline Systolic BP (mmHg) \\
\hline Diastolic BP (mmHg) \\
\hline Triglycerides $(\mathrm{mmol} / \mathrm{L})^{a}$ \\
\hline Total cholesterol (mmol/L) \\
\hline LDL-c (mmol/L) \\
\hline HDL-c (mmol/L) \\
\hline Fasting glucose (mmol/L) \\
\hline HOMA-IR \\
\hline $\operatorname{ALT}(U / L)$ \\
\hline AST (U/L) \\
\hline TBIL $(\mu \mathrm{mol} / \mathrm{L})$ \\
\hline DBIL $(\mu \mathrm{mol} / \mathrm{L})$ \\
\hline Free T3 $(\mathrm{pmol} / \mathrm{L})^{\mathrm{a}}$ \\
\hline Free T4 (pmol/L) \\
\hline Total T3 (nmol/L) ${ }^{a}$ \\
\hline Total T4 (nmol/L)a \\
\hline $\mathrm{TSH}(\mathrm{mlU} / \mathrm{L})^{\mathrm{a}}$ \\
\hline TRAb (IU/L) $)^{a}$ \\
\hline $\operatorname{TGAb}(I U / m L)^{a}$ \\
\hline TPOAb (IU/mL)a \\
\hline Serum Gpnmb (ng/mL) \\
\hline
\end{tabular}

\begin{tabular}{c}
\hline Before treatment \\
46 \\
$32 \pm 11$ \\
$34(73.9)$ \\
$4(8.5)$ \\
$20.3 \pm 2.8$ \\
$75.0 \pm 8.5$ \\
$43.3 \pm 14.3$ \\
$104 \pm 11$ \\
$123 \pm 16$ \\
$73 \pm 12$ \\
$0.87(0.71-1.06)$ \\
$3.72 \pm 0.84$ \\
$1.88 \pm 0.57$ \\
$1.42 \pm 0.42$ \\
$5.26 \pm 0.63$ \\
$1.63(1.13-2.57)$ \\
$36.8 \pm 15.0$ \\
$28.6 \pm 11.3$ \\
$13.9 \pm 7.6$ \\
$4.9 \pm 3.1$ \\
$22.4(15.3-100.2)$ \\
$67.2(40.2-82.0)$ \\
$7.6(5.3-12.3)$ \\
$379.0(268.8-387.0)$ \\
$0.006(0.004-0.009)$ \\
$18.9(10.3-29.4)$ \\
$39.7(12.5-323.2)$ \\
$41.8(6.0-192.4)$ \\
$47.6 \pm 8.6$ \\
\end{tabular}

\begin{tabular}{c}
\hline After treatment \\
\hline 46 \\
- \\
$3(6.4)$ \\
$21.4 \pm 2.7$ \\
$77.2 \pm 7.1$ \\
$13.8 \pm 11.7$ \\
$82 \pm 8$ \\
$117 \pm 14$ \\
$74 \pm 10$ \\
$1.05(0.74-1.33)$ \\
$5.40 \pm 0.99$ \\
$3.26 \pm 0.77$ \\
$1.62 \pm 0.33$ \\
$5.03 \pm 0.44$ \\
$1.68(1.14-1.93)$ \\
$22.3 \pm 13.0$ \\
$20.9 \pm 7.2$ \\
$12.1 \pm 4.4$ \\
$3.7 \pm 1.6$ \\
$4.6(3.8-5.4)$ \\
$13.1(11.6-15.4)$ \\
$1.6(1.3-2.2)$ \\
$94.6(79.8-111.0)$ \\
$0.487(0.008-3.663)$ \\
$8.4(2.2-17.7)$ \\
$22.3(13.0-152.5)$ \\
$52.2(5.8-299.2)$ \\
$36.8 \pm 5.5$ \\
\\
\hline
\end{tabular}

\begin{tabular}{|c|}
\hline P-value \\
\hline- \\
\hline- \\
\hline- \\
\hline$<0.001$ \\
\hline$<0.001$ \\
\hline$<0.001$ \\
\hline$<0.001$ \\
\hline$<0.001$ \\
\hline 0.001 \\
\hline 0.511 \\
\hline 0.130 \\
\hline$<0.001$ \\
\hline$<0.001$ \\
\hline 0.001 \\
\hline 0.013 \\
\hline 0.290 \\
\hline$<0.001$ \\
\hline$<0.001$ \\
\hline 0.050 \\
\hline 0.015 \\
\hline$<0.001$ \\
\hline$<0.001$ \\
\hline$<0.001$ \\
\hline$<0.001$ \\
\hline$<0.001$ \\
\hline$<0.001$ \\
\hline 0.949 \\
\hline 0.253 \\
\hline$<0.001$ \\
\hline
\end{tabular}

Data are presented as the mean \pm s.D. or median (interquartile range).

aAnalysis performed on log-transformed data.

ALT, alanine transaminase; AST, aspartate aminotransferase; BMR, basal metabolic rate; BP, blood pressure; DBIL, direct bilirubin; Gpnmb, glycoprotein non metastatic melanoma protein B; HOMA-IR, homeostasis model assessment of insulin resistance; LDL-c, low density lipoprotein cholesterol; TBIL, total bilirubin; T3, triiodothyronine; T4, thyroxine; TSH, thyroid stimulating hormone; TPOAb, thyroid peroxide antibody; TGAb, thyroglobulin antibody; TRAb, thyrotropin receptor antibody.

of thyroid antibodies including serum TRAb and TPOAb. Unfortunately, information regarding the association between circulating Gpnmb and autoimmune thyroid diseases (AITD) is largely unclear. Previous studies indicated that Gpnmb may regulate autoimmune responses through myeloid-derived suppressor cells or antigen presenting cells (APCs) $(20,21,22)$, and it is well established that both cell-mediated and humoral response contribute to the development of autoimmune thyroid diseases (AITD) $(23,24)$. Therefore, these observations suggest that Gpnmb may be involved in the autoimmune process of hyperthyroidism. To date, this study is the first clinical study to determine the role of circulating Gpnmb in the development of hyperthyroidism in humans. Our findings suggest that circulating Gpnmb concentrations might have prognostic significance in patients with hyperthyroidism.

This study provided an opportunity to investigate the role of circulating Gpnmb in patients with hyperthyroidism.
Nevertheless, there are several limitations to the present study. First, the study was based on case-control data with a limited sample size. Further studies should be replicated to determine the role of circulating Gpnmb in the general population. Second, it is impossible to determine the causal relationship between thyroid hormones and serum Gpnmb levels in the development of hyperthyroidism because of its cross-sectional design. Therefore, the causal association between circulating Gpnmb and hyperthyroidism should be evaluated in prospective cohort studies. Furthermore, the underlying mechanism of Gpnmb in the development of hyperthyroidism should be further studied in in vivo and in vitro.

In conclusion, our study provides clinical evidence for the first time revealing that circulating Gpnmb concentrations were markedly elevated in patients with hyperthyroidism and independently associated with risks of hyperthyroidism in relation to hypermetabolic state. https://ec.bioscientifica.com https://doi.org/10.1530/EC-20-0240 (c) 2020 The authors Published by Bioscientifica Ltd
This work is licensed under a Creative Commons Attribution-NonCommercial-NoDerivatives 4.0 enternationab dicense.ifica . com at 04/26/2023 01:29:53PM 
Table 5 Clinical correlation between changes of serum Gpnmb levels, and clinical and biochemical variables after treatment.

\begin{tabular}{|c|c|c|c|}
\hline Variables & $\beta$ & P-value & $\begin{array}{l}\text { Multiple } \\
\text { adjusted } \\
\text { P-value }\end{array}$ \\
\hline $\mathrm{BMI}\left(\mathrm{kg} / \mathrm{m}^{2}\right)$ & -0.031 & 0.844 & 0.543 \\
\hline Waist circumference $(\mathrm{cm})$ & -0.052 & 0.741 & 0.845 \\
\hline BMR (\%) & 0.477 & 0.001 & 0.002 \\
\hline Systolic BP (mmHg) & 0.214 & 0.175 & 0.145 \\
\hline Diastolic BP (mmHg) & 0.024 & 0.881 & 0.809 \\
\hline Total cholesterol (mmol/L) & 0.253 & 0.185 & 0.422 \\
\hline Total triglycerides (mmol/L) & 0.006 & 0.977 & 0.455 \\
\hline LDL-c (mmol/L) & 0.327 & 0.084 & 0.128 \\
\hline HDL-c (mmol/L) & -0.051 & 0.791 & 0.492 \\
\hline Fasting glucose (mmol/L) & 0.083 & 0.668 & 0.812 \\
\hline HOMA-IR & 0.272 & 0.154 & 0.288 \\
\hline $\operatorname{ALT}(U / L)$ & 0.111 & 0.553 & 0.755 \\
\hline AST (U/L) & 0.207 & 0.263 & 0.329 \\
\hline Free T3 (pmol/L) & 0.339 & 0.028 & 0.006 \\
\hline Free T4 (pmol/L) & 0.316 & 0.042 & 0.025 \\
\hline TSH (mIU/L) & -0.079 & 0.622 & 0.901 \\
\hline TRAb (IU/L) & 0.150 & 0.348 & 0.552 \\
\hline TGAb (IU/mL) & -0.091 & 0.598 & 0.688 \\
\hline TPOAb (IU/mL) & -0.023 & 0.891 & 0.894 \\
\hline
\end{tabular}

${ }^{a}$ Adjusted for age, gender, smoking, and BMI before treatment. $B M R$, basal metabolic rate; BP, blood pressure; LDL-c, low density lipoprotein cholesterol; HDL-c, high density lipoprotein cholesterol; HOMA-IR, homeostasis model assessment of insulin resistance; ALT, alanine aminotransferase; AST, aspartate aminotransferase; T3, triiodothyronine; T4, thyroxine; TSH, thyroid stimulating hormone; TPOAb, thyroid peroxide antibody; TGAb, thyroglobulin antibody; TRAb, thyrotropin receptor antibody.

These findings indicate that circulating Gpnmb may be involved in the thyroid hormone signaling network to modulate energy homeostasis and lipid metabolism and act as a prognostic factor in the development of hyperthyroidism in humans. However, the role of circulating Gpnmb in the development of hyperthyroidism needs to be further studied in vivo and in vitro.

\section{Supplementary materials}

This is linked to the online version of the paper at https://doi.org/10.1530/ EC-20-0240.

\section{Declaration of interest}

The authors declare that there is no conflict of interest that could be perceived as prejudicing the impartiality of the research reported.

\section{Funding}

This study was supported by the National Key Research and Development Project (Nos. 2018YFA0800404 and 2018YFC2001105), Natural Science Foundation and Key-Area Research and Development Program of Guangdong Province (No. 2018B030311031), Outstanding
Youth Development Scheme of Nanfang Hospital of Southern Medical University, and National Natural Science Foundation of China (No. 81970736).

\section{References}

1 Anyetei-Anum CS, Roggero VR \& Allison LA. Thyroid hormone receptor localization in target tissues. Journal of Endocrinology 2018 237 R19-R34. (https://doi.org/10.1530/JOE-17-0708)

2 Williams GR. Cloning and characterization of two novel thyroid hormone receptor beta isoforms. Molecular and Cellular Biology 2000 20 8329-8342. (https://doi.org/10.1128/mcb.20.22.8329-8342.2000)

3 Ribeiro MO, Carvalho SD, Schultz JJ, Chiellini G, Scanlan TS, Bianco AC \& Brent GA. Thyroid hormone - sympathetic interaction and adaptive thermogenesis are thyroid hormone receptor isoform - specific. Journal of Clinical Investigation 2001108 97-105. (https:// doi.org/10.1172/JCI12584)

4 Cheng SY, Leonard JL \& Davis PJ. Molecular aspects of thyroid hormone actions. Endocrine Reviews 201031 139-170. (https://doi. org/10.1210/er.2009-0007)

5 Fox CS, Pencina MJ, D'Agostino RB, Murabito JM, Seely EW, Pearce EN \& Vasan RS. Relations of thyroid function to body weight: cross-sectional and longitudinal observations in a community-based sample. Archives of Internal Medicine 2008168 587-592. (https://doi. org/10.1001/archinte.168.6.587)

6 Vaitkus JA, Farrar JS \& Celi FS. Thyroid hormone mediated modulation of energy expenditure. International Journal of Molecular Sciences 201516 16158-16175. (https://doi.org/10.3390/ ijms160716158)

7 Brent GA. Mechanisms of thyroid hormone action. Journal of Clinical Investigation 2012122 3035-3043. (https://doi.org/10.1172/ JCI60047)

8 Mullur R, Liu YY \& Brent GA. Thyroid hormone regulation of metabolism. Physiological Reviews 201494 355-382. (https://doi. org/10.1152/physrev.00030.2013)

9 Ross DS, Burch HB, Cooper DS, Greenlee MC, Laurberg P, Maia AL, Rivkees SA, Samuels M, Sosa JA, Stan MN, et al. 2016 American Thyroid Association guidelines for diagnosis and management of hyperthyroidism and other causes of thyrotoxicosis. Thyroid 201626 1343-1421. (https://doi.org/10.1089/thy.2016.0229)

10 Stefan N, Hennige AM, Staiger H, Machann J, Schick F, Krober SM, Machicao F, Fritsche A \& Haring HU. Alpha2-Heremans-Schmid glycoprotein/fetuin-A is associated with insulin resistance and fat accumulation in the liver in humans. Diabetes Care 200629 853-857. (https://doi.org/10.2337/diacare.29.04.06.dc05-1938)

11 Xiao F, Lin M, Huang P, Zeng J, Zeng X, Zhang H, Li X, Yang S, Li Z \& Li X. Elevated serum fibroblast growth factor 21 levels in patients with hyperthyroidism. Journal of Clinical Endocrinology and Metabolism 2015100 3800-3805. (https://doi.org/10.1210/jc.20151797)

12 Li M, Chen Y, Jiang J, Lu Y, Song Z, Zhang S, Sun C, Ying H, Fan X, Song Y, et al. Elevated serum neuregulin 4 levels in patients with hyperthyroidism. Endocrine Connections 20198 728-735. (https://doi. org/10.1530/EC-19-0175)

13 Ripoll VM, Irvine KM, Ravasi T, Sweet MJ \& Hume DA. Gpnmb is induced in macrophages by IFN-gamma and lipopolysaccharide and acts as a feedback regulator of proinflammatory responses. Journal of Immunology 2007178 6557-6566. (https://doi.org/10.4049/ jimmunol.178.10.6557)

14 Singh M, Del Carpio-Cano F, Belcher JY, Crawford K, Frara N, Owen TA, Popoff SN \& Safadi FF. Functional roles of osteoactivin in normal and disease processes. Critical Reviews in Eukaryotic Gene Expression 201020 341-357. (https://doi.org/10.1615/ critreveukargeneexpr.v20.i4.50) 
15 Frara N, Abdelmagid SM, Sondag GR, Moussa FM, Yingling VR, Owen TA, Popoff SN, Barbe MF \& Safadi FF. Transgenic expression of osteoactivin/gpnmb enhances bone formation in vivo and osteoprogenitor differentiation ex vivo. Journal of Cellular Physiology 2016231 72-83. (https://doi.org/10.1002/jcp.25020)

16 Oyewumi MO, Manickavasagam D, Novak K, Wehrung D, Paulic N, Moussa FM, Sondag GR \& Safadi FF. Osteoactivin (GPNMB) ectodomain protein promotes growth and invasive behavior of human lung cancer cells. Oncotarget 20167 13932-13944. (https:// doi.org/10.18632/oncotarget.7323)

17 Haralanova-Ilieva B, Ramadori G \& Armbrust T. Expression of osteoactivin in rat and human liver and isolated rat liver cells. Journal of Hepatology 200542 565-572. (https://doi.org/10.1016/j. jhep.2004.12.021)

18 Gong X-M, Li Y-F, Luo J, Wang J-Q, Wei J, Wang J-Q, Xiao T, Xie C, Hong J, Ning G, et al. Gpnmb secreted from liver promotes lipogenesis in white adipose tissue and aggravates obesity and insulin resistance. Nature Metabolism 20191 570-583. (https://doi org/10.1038/s42255-019-0065-4)

19 Katayama A, Nakatsuka A, Eguchi J, Murakami K, Teshigawara S, Kanzaki M, Nunoue T, Hida K, Wada N, Yasunaka T, et al. Beneficial impact of Gpnmb and its significance as a biomarker in nonalcoholic steatohepatitis. Scientific Reports 20155 16920. (https://doi. org/10.1038/srep16920)

20 Chung JS, Dougherty I, Cruz Jr PD \& Ariizumi K. Syndecan-4 mediates the coinhibitory function of DC-HIL on T cell activation. Journal of Immunology 2007179 5778-5784. (https://doi.org/10.4049/ jimmunol.179.9.5778)

21 Chung JS, Sato K, Dougherty II, Cruz PD \& Ariizumi K. DC-HIL is a negative regulator of T lymphocyte activation. Blood $2007 \mathbf{1 0 9}$ 4320-4327. (https://doi.org/10.1182/blood-2006-11-053769)

22 Chung J-S, Tamura K, Akiyoshi H, Cruz Jr PD \& Ariizumi K. The DC-HIL/syndecan-4 pathway regulates autoimmune responses through myeloid-derived suppressor cells. Journal of Immunology 2014 192 2576-2584. (https://doi.org/10.4049/jimmunol.1301857)

23 Antonelli A, Ferrari SM, Corrado A, Di Domenicantonio A \& Fallahi P. Autoimmune thyroid disorders. Autoimmunity Reviews 2015 14 174-180. (https://doi.org/10.1016/j.autrev.2014.10.016)
24 Kristensen B. Regulatory B and T cell responses in patients with autoimmune thyroid disease and healthy controls. Danish Medical Journal 201663 B5177.

25 Gale A. Estimation of the basal metabolic rate from formulae based on pulse-rate and pulse pressure. Lancet 1931217 1287-1288. (https://doi.org/10.1016/S0140-6736(00)49092-6)

26 Friedewald WT, Levy RI \& Fredrickson DS. Estimation of the concentration of low-density lipoprotein cholesterol in plasma, without use of the preparative ultracentrifuge. Clinical Chemistry 197218 499-502. (https://doi.org/10.1093/clinchem/18.6.499)

27 Matthews DR, Hosker JP, Rudenski AS, Naylor BA, Treacher DF \& Turner RC. Homeostasis model assessment: insulin resistance and beta-cell function from fasting plasma glucose and insulin concentrations in man. Diabetologia 198528 412-419. (https://doi. org/10.1007/BF00280883)

28 Wallace TM, Levy JC \& Matthews DR. Use and abuse of HOMA modeling. Diabetes Care 200427 1487-1495. (https://doi. org/10.2337/diacare.27.6.1487)

29 Hashimoto K, Cohen RN, Yamada M, Markan KR, Monden T, Satoh T, Mori M \& Wondisford FE. Cross-talk between thyroid hormone receptor and liver $\mathrm{X}$ receptor regulatory pathways is revealed in a thyroid hormone resistance mouse model. Journal of Biological Chemistry 2006281 295-302. (https://doi.org/10.1074/jbc. M507877200)

30 Hashimoto K, Yamada M, Matsumoto S, Monden T, Satoh T $\&$ Mori M. Mouse sterol response element binding protein-1c gene expression is negatively regulated by thyroid hormone. Endocrinology 2006147 4292-4302. (https://doi.org/10.1210/ en.2006-0116)

31 Liu YY, Heymann RS, Moatamed F, Schultz JJ, Sobel D \& Brent GA. A mutant thyroid hormone receptor alpha antagonizes peroxisome proliferator-activated receptor alpha signaling in vivo and impairs fatty acid oxidation. Endocrinology 2007148 1206-1217. (https://doi. org/10.1210/en.2006-0836)

32 Liu YY \& Brent GA. Thyroid hormone crosstalk with nuclear receptor signaling in metabolic regulation. Trends in Endocrinology and Metabolism 201021 166-173. (https://doi.org/10.1016/j. tem.2009.11.004)

Received in final form 30 June 2020

Accepted 19 July 2020

Accepted Manuscript published online 20 July 2020 https://ec.bioscientifica.com https://doi.org/10.1530/EC-20-0240 (c) 2020 The authors Published by Bioscientifica Ltd
This work is licensed under a Creative Commons Attribution-NonCommercial-NoDerivatives 4.0 Internationab bicense ifica . com at $04 / 26 / 2023 \quad 01: 29: 53 \mathrm{PM}$ 